\title{
Cotton-wool spots and retinal light sensitivity in diabetic retinopathy
}

Toke Bek, Henrik Lund-Andersen

\begin{abstract}
In 14 eyes of 14 patients with diabetic retinopathy the light sensitivity of retinal cottonwool spots was studied by computerised perimetry, and the visual field data were accurately correlated with the corresponding morphology as seen on fundus photographs and fluorescein angiograms. In 12 of the eyes the examinations were repeated within one year in order to follow changes in retinal light sensitivity during the evolution of the lesions. Retinal cotton-wool spots were in all eyes associated with localised non-arcuate scotomata in the visual field. In four eyes the cottonwool spots disappeared within three months of the first examination, and in two of these cases the corresponding scotomata disappeared together with the morphological lesions. In eight eyes the cotton-wool spots (and the corresponding scotomata) had not resolved one year after the first examination. The mean blood pressure showed no significant difference between the patients in whom the lesions resolved within three months and the patients in whom the lesions persisted longer.

The retinal cotton-wool spot is a frequent finding in a variety of diseases which involve the ocular fundus. ' In diabetic retinopathy the lesion may be among the initial signs of the disease, and when the lesions are numerous they may indicate
\end{abstract}

Ophthalmology,

University of

Copenhagen, Gentofte

Hospital, 2900 Hellerup,

Denmark

T Bek

$H$ Lund-Andersen

Correspondence to:

Toke Bek, MD.

Accepted for publication

30 July 1990

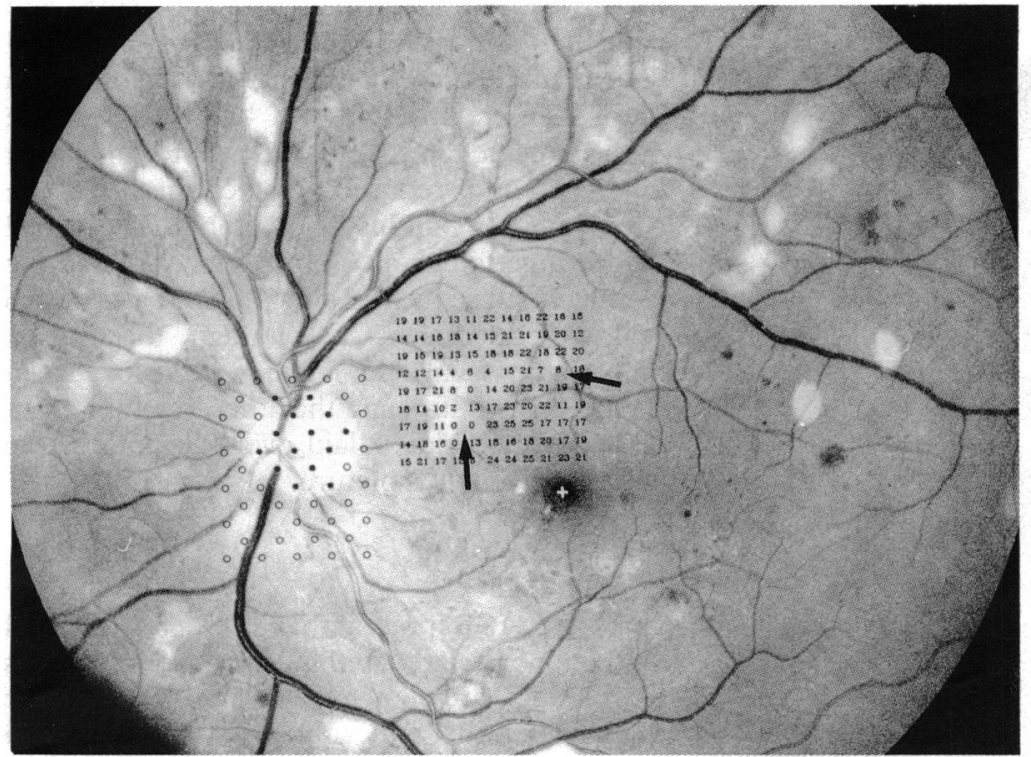

Figure $1 A$ A left eye fundus photograph of a patient with diabetic retinopathy dominated by cotton-wool spots. The perimetric stimulus pattern extended from degree co-ordinates $(x, y)=(-$ $9,-10)$ to $(1,-2)$, and the vistal field data have been superimposed on to the corresponding fundus morphology. Two areas with low decibel values are seen to correlate with retinal cottonwool spots (arrows). The low decibel values are predominantly located on the peripheral aspect of the lesions. rapidly progressing retinopathy. ${ }^{2}$ Although it is known that the cotton-wool spot can be caused by focal retinal ischaemia, no single explanation has been given which consistently accounts for the aetiology of the lesion in all cases. ${ }^{3}$

The retinal cotton-wool spot has been mainly investigated by photographic and histological techniques which demonstrate the morphological aspects of the lesion. The inclusion of techniques for the assessment of neurosensory function can be expected to supplement our understanding of its pathophysiology. In a prior study the visual field of six diabetic patients with retinal cotton-wool spots was examined by a manual perimetric technique, ${ }^{4}$ but the exact correlation between visual field data and the corresponding fundus morphology was not accounted for, and the visual field findings were not systematically followed during the evolution of the lesions.

In the present study the light sensitivity of retinal areas with cotton-wool spots was examined by computerised perimetry, and by a newly developed optical technique the visual field data were accurately correlated with the corresponding fundus morphology as seen on fundus photographs and fluorescein angiograms. The examinations were repeated within one year in order to follow changes in retinal light sensitivity during the evolution of the lesions.

\section{Materials and methods}

\section{SUBJECTS}

Fourteen eyes of 14 patients with insulin dependent diabetes mellitus in which retinal cottonwool spots had been detected by funduscopic examination were subjected to computerised perimetry, and the visual field data were accurately correlated with the corresponding morphology as seen on fundus photographs and fluorescein angiograms. The patients underwent routine ophthalmic examinations, including visual acuity, slit-lamp examination, tonometry, ophthalmoscopy, fundus photography, and fluorescein angiography. Their informed consent was obtained. The blood pressure was measured at the initial examination or was obtained from medical records within two months of the initial examination. The ametropia of the eyes ranged between $-2.0 \mathrm{D}$ and $+1.25 \mathrm{D}$, and the most severe astigmatism was $1.5 \mathrm{DC}$. Visual acuity was $6 / 9$ in one patient and $6 / 6$ or better in all the other patients.

Except for funduscopic signs of retinopathy the other part of the ophthalmological examination was normal in all the patients included. In 12 eyes the examinations were repeated within three months of the first examination and, in cases 


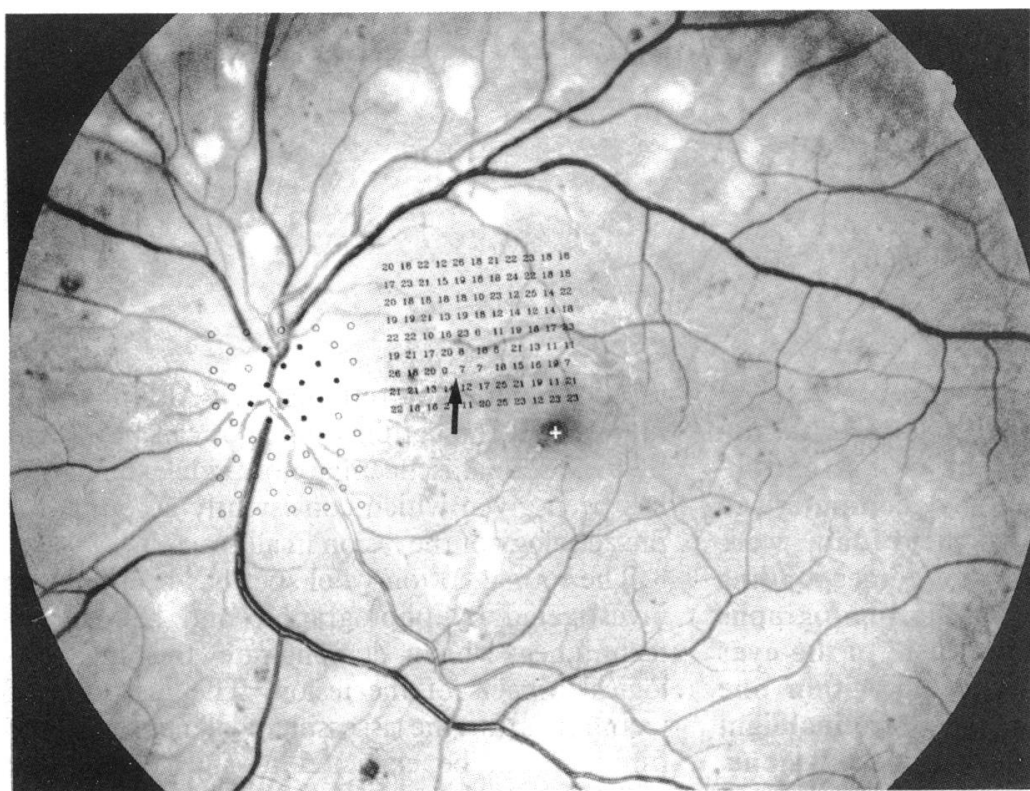

Figure $1 B \quad A$ fundus photograph of the same eye three months later. The cotton-wool spots which were studied by perimetry have now resolved, but an area with low decibel values persists where one of the cotton-wool spots was located (arrow). Another cotton-wool spot has now appeared below the examined area.

where the cotton-wool spots persisted, again one year after the initial examination.

\section{PHOTOGRAPHY}

Photography was performed with a Canon CF$60 Z$ fundus camera employing Ektachrome 64 (EPR 135-36) film for fundus photography, and Ilford HP-5 black-and-white film for fluorescein

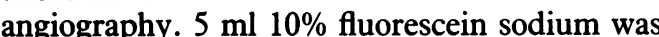
injected into an antecubital vein for fluorescein angiography. Angiograms were taken of the central fundus in fast sequence during the filling phase of the vessels, and at regular intervals until $15 \mathrm{~min}$ after injection. Prior to photography cycloplegia and mydriasis were induced with metaoxedrine $10 \%$ and tropicamide $1 \%$ eyedrops.

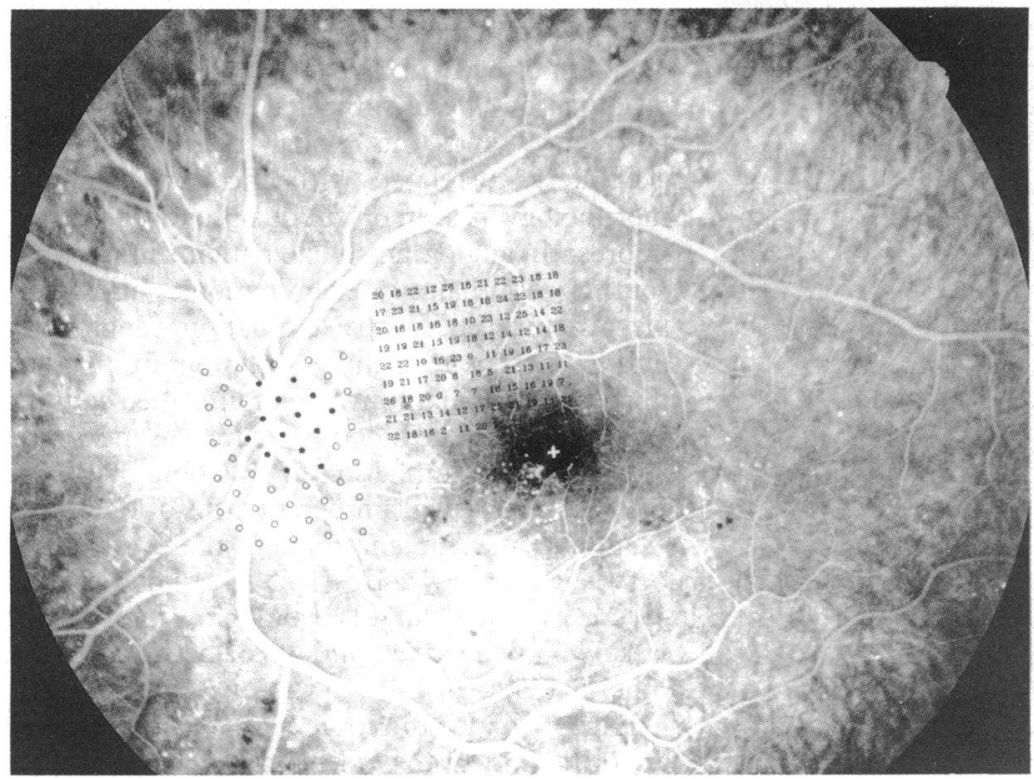

Figure 1C A fluorescein angiogram of the same eye obtained on the same day as Fig $1 B$, and visual field data have been superimposed. The retinal areas corresponding to the persisting scotoma shows some pathology, mainly as focal leakage of fluorescein.

\section{PERIMETRY}

Perimetry was carried out with a Humphrey Field Analyzer (HFA). Full-threshold examinations were performed in stimulus patterns with a density of $1^{\circ}$ employing Goldmann stimulus size I. These were the best parameters available on the HFA for the detection of small scotomata. ${ }^{5}$ Since the minimum density of customised stimulus patterns on the HFA is $2^{\circ}$, four intersecting patterns with a displacement of $1^{\circ}$ in the vertical and the horizontal plane were subsequently tested, and finally merged. The stimulus patterns were placed in areas of the visual field that approximately corresponded to the retinal areas where morphological lesions had been observed by prior funduscopic examination.

Threshold values that deviated more than $5 \mathrm{~dB}$ from the expected value calculated on the basis of answers from adjoining points were retested (standard procedure on the HFA). The blind spot was delimited with Goldmann stimulus size II with a single intensity of $10 \mathrm{~dB}$ in two special point patterns located in the area of the blind spot, one (pattern A) extending from degree coordinates $(x, y)=(11,-7)$ to $(19,3)$ and one (pattern B) from degree co-ordinates $(x, y)=$ $(12,-8)$ to $(18,2)$. By merging these two patterns a spatial resolution of $1.4^{\circ}$ could be obtained in this area. All patients could see the perimeter screen sharply during perimetry without the addition of a corrective lens. Fixation was continuously checked on the fixation monitor supplied on the HFA, which displays the eye on a video screen during perimetry. Fixation was stable during all test sessions in all the patients included.

\section{SUPERIMPOSITION TECHNIQUE}

The superimposition of visual field data on to fundus pictures was carried out according to a technique previously described. ${ }^{6}$ This technique is applicable to a fundus photograph (subtending approximately $60^{\circ}$ with the camera employed here) with the foveal region in the centre and with the accuracy of the overlay or visual field data on to the corresponding fundus photograph more than $1^{\circ}$. Briefly, with knowledge of the optical imaging in the fundus camera, the visual field printout is reduced to match the size of the corresponding fundus photograph. The visual field is inverted about a horizontal axis, and the overlay is subsequently done on the basis of two points of reference. One of them, the fixation point in the visual field, is laid over the foveola on the fundus picture, and the other, the blind spot in the visual field, is laid over the optic nerve head on the fundus picture.

\section{Results}

In Figs 1 to 3 representative examples of visual field data correlated with corresponding fundus morphology are shown. The numbers indicate threshold decibel values obtained with Goldmann stimulus size I, and each number has been superimposed on to the corresponding fundus morphology. In points where the threshold has been tested twice, the average of the two decibel values has been employed. The fixation point is 


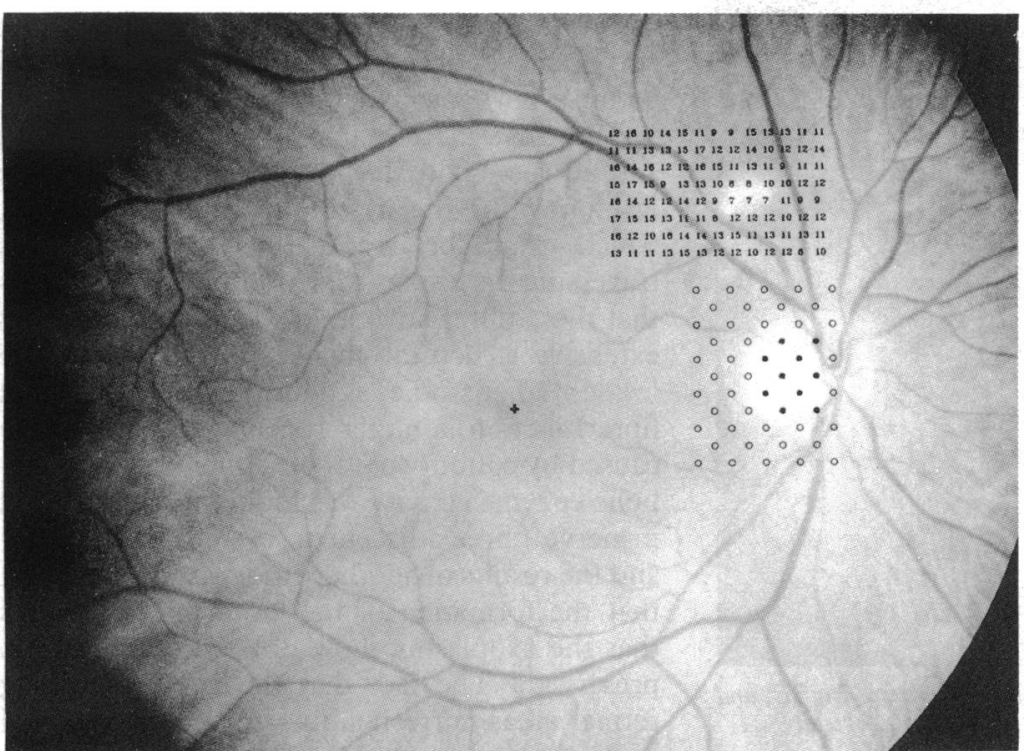

Figure 2A A right eye fundus photograph of a patient with insulin dependent diabetes mellitus. The only sign of retinopathy is a cotton-wool spot located above the optic nerve head. The perimetric stimulus pattern extended from degree co-ordinates $(x, y)=(6,-16)$ to $(18,-9)$. The visual field data have been superimposed on to the corresponding fundus morphology, and low decibel values ( a relative scotoma) have been superimposed on to the cotton-wool spot.

indicated with a cross. In the screening tests employed to delimit the blind spot open circles indicate seen points, whereas closed circles indicate points not seen.

In all 14 eyes examined the retinal cotton-wool spots were associated with localised scotomata in the visual field which appeared non-arcuate within the area of the visual field tested. In five of these cases the non-functioning retinal area corresponding to the scotoma extended beyond the peripheral aspect of the morphological lesion (see Fig 1), a finding which could not be explained as a result of inaccuracies of the superimposition technique.

Twelve eyes were re-examined. In four eyes the lesion had disappeared within three months

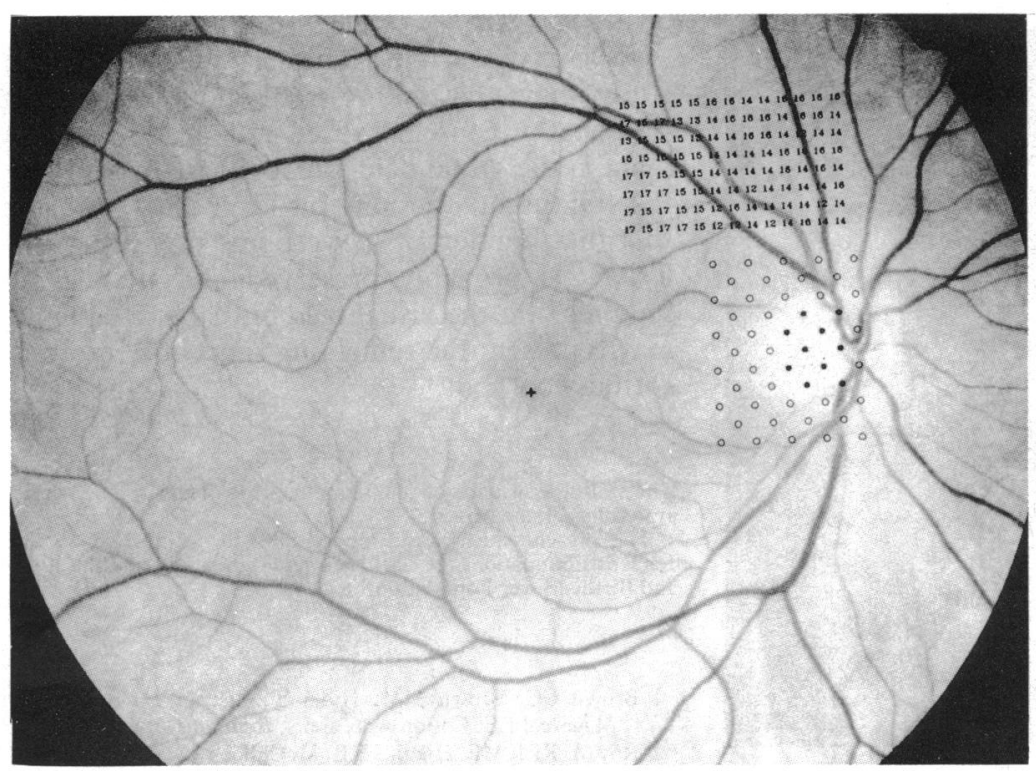

Figure 2B A fundus photograph of the same eye three months later. Visual field data obtained at that time are superimposed. The cotton-wool spot has now entirely disappeared, and no scotoma is detected in the visual field. of the initial examination. In two of these cases the scotoma and some angiographical pathology corresponding to the now resolved cotton-wool spot persisted (Fig 1). In the other two cases the scotoma had disappeared together with the funduscopic lesion, and no angiographic traces of the lesion persisted (Fig 2). In eight eyes the cotton-wool spots, though changed in appearance, were still present together with the corresponding visual field scotomata one year from the initial examination (Fig 3).

There was no significant difference between the mean blood pressure of the patients in whom cotton-wool spots resolved within three months - systolic mean, 155 (range 140-170) $\mathrm{mmHg}$, diastolic mean, 88 (range 75-95) $\mathrm{mmHg}$ - and the blood pressure of the patients in whom the lesions persisted longer - systolic mean, 148 (range 120-175) $\mathrm{mmHg}$, diastolic mean, 90 (range 70-100) $\mathrm{mmHg}$.

\section{Discussion}

The findings of the present study considerably extend the findings ${ }^{4}$ from a quantitative perimetric technique and an accurate technique for superimposing visual field data on to the corresponding fundus morphology. Retinal cottonwool spots were consistently associated with localised non-arcuate scotomata in the visual field. Of 12 eyes which were re-examined the cotton-wool spots had disappeared within a few months in four, and in two of these the corresponding scotoma disappeared together with the morphological lesion. In the other eight eyes the cotton-wool spots (and the corresponding scotomata) persisted for more than one year.

Retinal cotton-wool spots are defined on the basis of their funduscopic appearance; typically they are white fluffy areas of retinal opacity with feathery margins. This appearance is assumed to reflect focal swelling of the nerve fibres, which is due to intracellular fluid and organelles accumulated secondary to interrupted axoplasmic flow. The aetiology of axonal disturbance at the site of the lesion is controversial, and no single explanation consistently explains the available data. ${ }^{3}$ It is generally believed, however, that focal retinal ischaemia is often a factor in its pathogenesis.

The natural history of the cotton-wool spots followed in the present study showed two different patterns. In one group of patients the lesions resolved within three months of the initial examination, whereas in another group the lesions persisted for more than one year. Since the age of the cotton-wool spots at the time of the initial examination is unknown, it is uncertain whether the lifetime of the lesions actually differed. If it did, however, the observed difference in natural history of the lesions might perhaps reflect differences in their pathophysiological basis. This view is supported by studies which have shown the lifetime of cotton-wool spots to vary considerably in different retinal diseases, such as for example hypertensive and diabetic retinopathy. ${ }^{89}$ The fact that no difference was found between the mean blood pressure in the two groups of patients, however, argues against raised blood pressure as a cause of the lesions in one of these groups. This view finds 


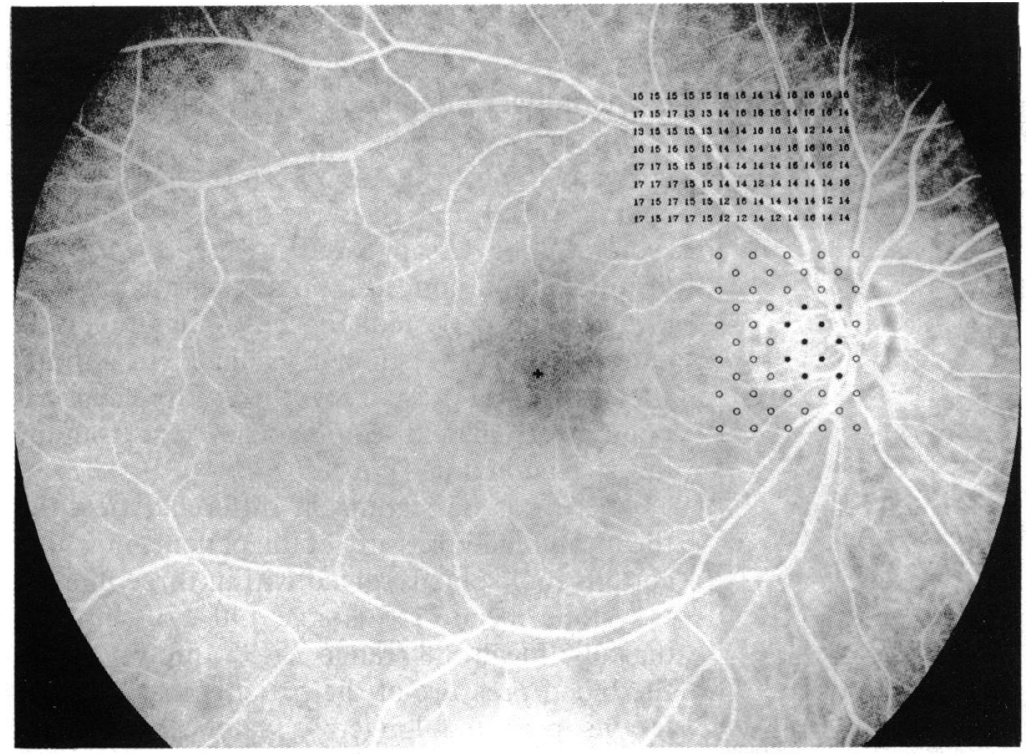

Figure 2C A fluorescein angiogram of the same eye obtained on the same day as Fig $3 B$, and visual field data have been superimposed. No angiographic traces of the funduscopically resolved cotton-wool spot can be seen.

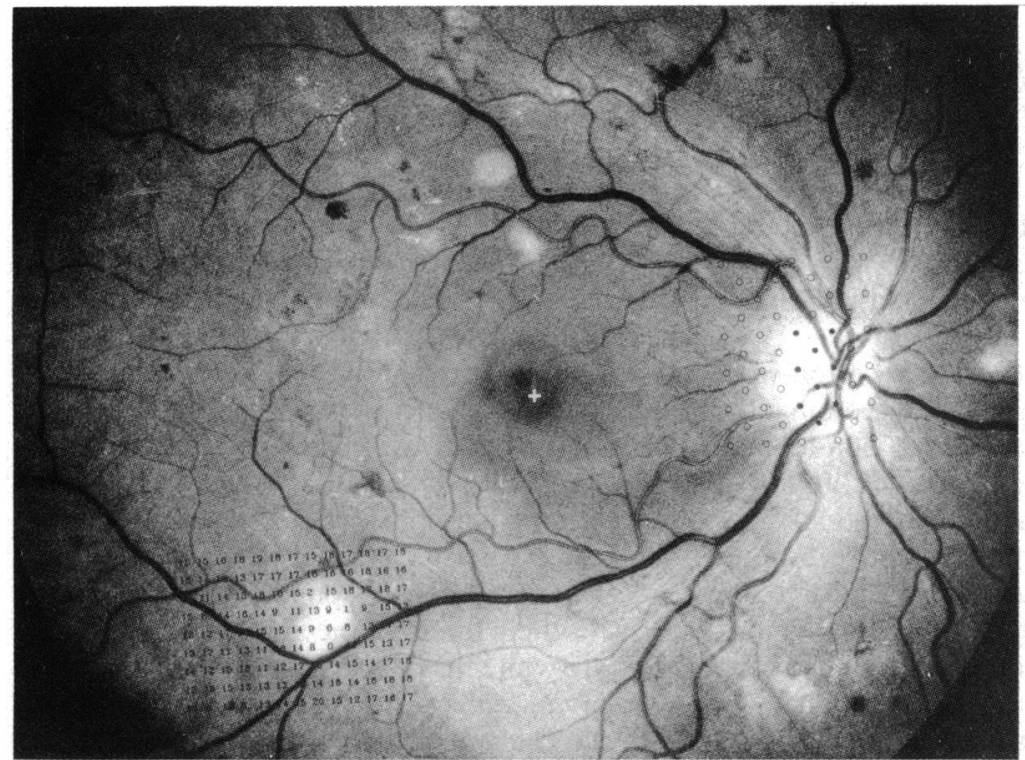

Figure $3 A \quad$ A right eye fundus photograph of a diabetic patient with background retinopathy of heterogeneous morphology. The perimetric stimulus pattern extended from degree co-ordinates $(x, y)=(-20,8)$ to $-8,16)$. The visual field data have been superimposed on to the corresponding fundus morphology, and a scotoma in the visual field is seen to correspond to a retinal cotton-wool spot at the lower temporal branch.

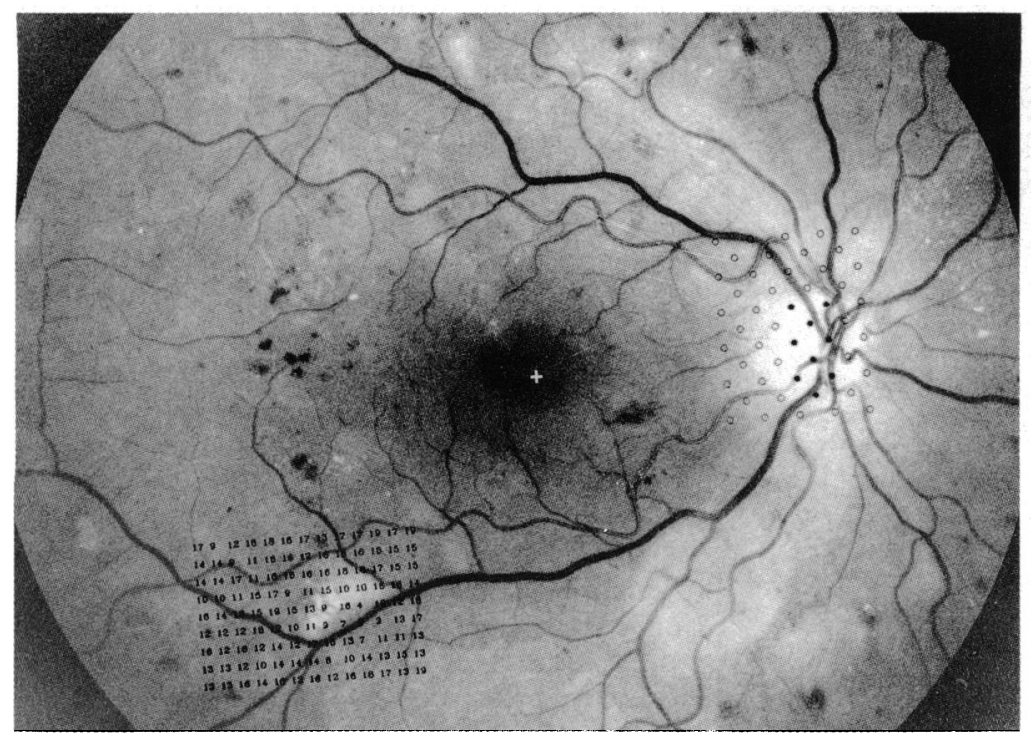

Figure $3 B \quad A$ fundus photograph of the same eye 12 months later. The retinal cotton-wool spot (though changed in appearance) is seen to persist together with a scotoma in the visual field (l(n) decibel values). Other cotton-wool spots along the upper temporal arch and nasal to the optic nerve head (compare with Fig $3 A$ ) have now disappeared.

further support in a prior study that showed no correlation between blood pressure and retinal cotton-wool spots in diabetic retinopathy. ${ }^{10}$

The scotomata found in association with cotton-wool spots did not extend in an arcuate fashion within the area of the visual field tested, which would be expected if the nerve fibres traversing the lesions were injured. It thus seems that these fibres are still viable despite probably extensive structural changes at the site of the lesion. Other studies support the idea that nerve fibre function is rather resistant to the damage caused by cotton-wool spots. In one study metabolic enzyme activity was found to be preserved in nerve fibres adjacent to cotton-wool spots, " and the results of another study strongly suggest that the formation of the lesion itself requires that the axoplasmic flow of the nerve fibres is preserved.' The fact that the non-functioning retinal areas corresponding to the cotton-wool spots in some cases extended beyond the peripheral aspects of the lesion remains to be explained. The finding, however, could perhaps be a parallel to evidence from experimentally produced cotton-wool spots showing that axons may penetrate some way into the lesion before swelling is seen histologically.

The fact that some scotomata persisted after resolution of the cotton-wool spots probably indicates that the recorded visual loss is not always entirely due to opacity of the lesion blocking the perimetric stimulus light from reaching the retinal photoreceptors, but probably is also due to structural injury to some part of the retina. This injury is probably reflected in the persisting angiographic pathology at the site of the resolved cotton-wool spot in these cases. In some studies it has been reported that scotomata sometimes occur in the visual field of diabetic patients without any lesion in the fundus. ${ }^{1213}$ These scotomata have been interpreted as an indication that neurosensory cell damage occurs before the development of morphological lesions in this disease. This interpretation, however, may easily be wrong if the scotomata represent the remaining signs of a resolved cotton-wool spot.

In conclusion, the findings of the present study show that cotton-wool spots of diabetic retinopathy cause localised non-arcuate scotomata in the visual field which may persist when the funduscopic lesions resolve. They indicate that the function of nerve fibres traversing the lesions is probably preserved and that small scotomata in the visual field of diabetic patients may represent the remaining signs of a resolved cotton-wool spot.

The skilful assistance of photographer Hans Henrik Petersen is gratefully acknowledged.

The present study was supported by the Mimi and Victor Larsens Foundation, the Carl Larsens Foundation, and the John and Birthe Meyer Foundation.

1 Brown GC, Brown MM, Hiller T, Fischer D, Benson E, Magargal LE. Cotton-wool spots. Retina 1985; 5: 206-14. 2 Roy M, Rick ME, Higgins KE, McCulloch JC. Retinal cottonwool spots: an early finding in diabetic retinopathy? $\mathrm{Br} \mathcal{F}$ Ophthalmol 1986; 70: 772-8.

3 Ashton N. Pathophysiology of retinal cotton-wool spots. Br Med Bull 1970; 26: 143-50. 
4 Williams DK, Drance S, Harris GS, Fairclough M. Diabetic cotton wool spots: an evaluation using perimetric and angiographic techniques. Can f Ophthalmol 1970; 5: 68-77.

5 Bek T, Lund-Andersen $H$. The influence of stimulus size on perimetric detection of small scotomata. Graefes Arch Clin Exp Ophthalmol 1989; 227: 531-4.

6 Bek T, Lund-Andersen H. Accurate superimposition of visua field data onto fundus pictures. Acta Ophthalmol (Kbh) 1990 ; 69: 11-8.

7 McLeod D, Marshall J, Kohner EM, Bird A. The role of axoplasmic transport in the pathogenesis of retinal cottonwool spots. Br f Ophthalmol 1977; 61: 177-91.

8 Hodge JV, Dollery CT. Retinal soft exudates. A clinical study by colour and fluorescence photography. $Q \mathcal{F}$ Med 1964; 129 (N Ser 33): 117-31.
9 Kohner EM, Dollery CT, Bulpitt CJ. Cotton-wool spots in diabetic retinopathy. Diabetes 1969; 18: 691-704.

10 Esmann V, Lundbaek K, Madsen PH. Types of exudates in diabetic retinopathy. Acta Med Scand 1963; 174: 37584.

11 Diezel PB, Willert HG. Morphologie und Histochemie der Harten und weichen Exsudate der Retina bei Diabetes mellitus und essentieller Hypertonie. Klin Monatsbl Augenheilkd 1961; 139: 475-91.

12 Bloom A, Heath JH, Kelsey PR, Hunter PR, Bridgden WD. The use of the Roth-Keeler central field scotometer in the study of diabetic retinopathy. Ophthalmic Res 1972; 3: 16673.

13 Roth JA. Central visual field in diabetes. $\mathrm{Br} \mathcal{F}$ Ophthalmol 1969; 53; 16-25. 